\title{
La doctrina social católica en el proceso industrializador de la España franquista: el caso del grupo farmacéutico Alter
}

\author{
Raúl Rodríguez Nozal (*) y Antonio González Bueno (**) \\ (*) orcid.org/0000-0003-2565-4695. Unidad Docente de Salud Pública, Medicina Legal e \\ Historia de la Ciencia. Universidad de Alcalá. raulrn@uah.es \\ (**) orcid.org/0000-0002-1933-4620. Departamento de Farmacia y Tecnología Farmacéutica. \\ Universidad Complutense de Madrid. agbueno@ucm.es
}

Dynamis

[0211-9536] 2015; 35 (2): 433-457

http://dx.doi.org/10.4321/S0211-95362015000200008
Fecha de recepción: 24 de diciembre de 2013

Fecha de aceptación: 24 de noviembre de 2014

SUMARIO: 1.-Paternalismo industrial y doctrina social católica. 2.-Juan José Alonso Grijalba (1894-1962). 3.-Los Laboratorios Alter: un vivero para la materialización de la doctrina social católica en pleno franquismo. 3.1.-El componente económico. 3.2.-El componente culturalrecreativo. 3.3.-El componente moral-religioso. 4.-Corolario.

RESUMEN: Los Laboratorios Alter, y el grupo de empresas generadas en torno a Juan José Alonso Grijalba (1894-1962) durante el franquismo, tuvieron como fundamento empresarial la doctrina social católica, de la que este farmacéutico fue firme defensor y propagandista. En este trabajo esbozamos la biografía de este empresario, exponemos sus principios ideológicos y analizamos el modo en que tales teorías se pusieron en práctica, en los Laboratorios Alter, desde tres aspectos: económico, cultural-recreativo y moral-religioso. La actuación empresarial que traslucen los escritos de Juan José Alonso son los de un «patronazgo patriarcal», su aspiración parece la de convertir Alter en una «fábrica-convento» bajo las bases programáticas del humanismo católico, en las que el empresario asume un rol claramente despótico, y en la que la intervención del obrero se reduce a aceptar las normas - y las prebendas - ofrecidas por el empresario.

PALABRAS CLAVE: Franquismo, industria farmacéutica, doctrina social católica, Juan José Alonso Grijalba, Laboratorios Alter.

KEY WORDS: Francoist Spain, pharmaceutical industry, Catholic social doctrine, Juan José Alonso Grijalba, Alter Laboratories. 


\section{Paternalismo industrial y doctrina social católica $\left(^{*}\right)$}

El término «paternalismo industrial» ha venido utilizándose para referirse a ciertas prácticas empresariales en las que los patronos tratan de mejorar las condiciones de vida del obrero, más allá de lo legalmente establecido en materia de salario y de condiciones laborales. Tuvieron su apogeo, en la mayor parte de los países industrializados, durante la segunda mitad del XIX y las dos primeras décadas del XX y, en España, resurgirían a partir de los años cuarenta, con la dictadura franquista ${ }^{1}$.

El paternalismo industrial, también conocido bajo los términos de «subsidiarismo empresarial»o «feudalismo industrial» ${ }^{2}$, aparece por conveniencias meramente pragmáticas; la clave era evitar cualquier atisbo de autoorganización obrera y mantener la dominación fuera de la fábrica a través de actuaciones - viviendas, comedores, seguros sociales, etc.-, aparentemente de tinte humanitario, nacidas del deber social de las clases poderosas hacia las más desfavorecidas. Con la irrupción del socialismo, este

(*) El presente trabajo ha sido financiado con cargo al proyecto de investigación HAR2013-42536-P del Ministerio de Economía y Competitividad.

1. Alemany García, Macario. El concepto y la justificación del paternalismo [Tesis doctoral, dirigida por Manuel Atienza y Juan Ruiz Manero]. Alicante: Universidad de Alicante; 2005, p. 11-12. Sobre la implantación de los mecanismos característicos del paternalismo industrial en algunas empresas españolas véanse los estudios de: Bogaerts, Jorge. El mundo social de ENSIDESA: estado y paternalismo industrial (1950-1973). Avilés: Azucel; 2000; García García, José Luis. Prácticas paternalistas. Un estudio antropológico sobre los mineros asturianos. Barcelona: Ariel; 1996; Peña Rambla, Fernando. Paternalismo y control social en la industria franquista: la empresa Segarra de la Vall d'Uixó. Sociología del Trabajo. 1998; 34: 109-132; Viruela Martínez, Rafael. Organización del trabajo y diferencias de género en la industria del calzado durante la autarquía: el caso de la empresa Segarra de la Vall d'Uixó. Scripta Nova, [artículo en Internet]. 2002 [citado 15 Dic 2013]; 6 (119) [http://www.ub.edu/geocrit/sn/ sn119-82.htm]; Payá Rico, Andrés. Formació professional, instrucció i adoctrinament a l'empresa Segarra (la Vall d'Uixó, Castelló) durant el franquisme. Educació i Història - Revista d'Història de l'Educació. 2010; 15: 229-252; Maira Vidal, María del Mar. El paternalismo industrial en el régimen franquista, la colonia industrial Ciudad Pegaso en Madrid. Sociología del Trabajo. 2009; 65: 137-162; Rodríguez Galdo, María Xosé; Losada Álvarez, Abel. Paternalismo empresarial y 'desarrollismo'. Reflexiones sobre la construcción del poblado minero de Fontao. Revista Galega de Economía. 2007; 16 (extraordinario): 117-138; Alonso del Val, Ignacio; Andrés Gómez, Valentín. De obrero a productor: Standard Eléctrica. Un ejemplo de paternalismo industrial durante el franquismo. In: Trujillano Sánchez, José Manuel; Gago González, José María, eds. Historia y Fuentes Orales. «Historia y Memoria del Franquismo» [Actas IV Jornadas. Ávila, Octubre 1994]. Ávila: Fundación Cultural Santa Teresa; 1997, p. 503-519.

2. Sierra Álvarez, José. El obrero soñado. Ensayo sobre el paternalismo industrial (Asturias, 18601917). Madrid: Siglo XXI; 1990, p. 75. 
paternalismo industrial, de talante práctico, sufrió un importante deterioro que le llevó a una ideologización más acusada, defendida sobre todo por los pensadores católicos belgas y franceses - como Fréderic Le Play (18061882) - , de marcada influencia en los católicos sociales españoles, y cuyo sustento ideológico fueron las encíclicas Rerum Novarum (1891) de León XIII y Quadragesimo Anno (1931) de Pío XI.

La feroz represión ideológica impulsada por el régimen de Franco, tras finalizar la Guerra Civil, sojuzgó cualquier atisbo de conflictividad obrera y propició un rebrote de las prácticas paternalistas lideradas o tuteladas por el colectivo católico, apoyadas - sin tapujos - por el Estado franquista. Las bases doctrinales de este movimiento fueron elaboradas antes de la Guerra Civil y se fundamentan en la supeditación de la economía a la moral ${ }^{3}$. Pese a que las actuaciones paternalistas en la España franquista estuvieron lideradas por la Iglesia y por la derecha tradicional, generalmente a través de empresarios con fuertes convicciones religiosas, no debemos pasar por alto la implicación de Falange en las iniciativas paternalistas estatales ${ }^{4}$; de hecho El Fuero del Trabajo (1938) fue un híbrido entre dos conceptos de Estado: el corporativo-tradicionalista, apoyado por carlistas y católicos, y el corporativo-fascista, defendido por Falange Española y de las JONS ${ }^{5}$.

En este sentido, durante los primeros años del franquismo se publicaron algunas disposiciones de carácter paternalista, muy cómodas para el Régimen por cuanto trasladaban ciertas obligaciones a los empresarios que, de otra manera, hubieran tenido que ser asumidas por el Estado: un decreto de 8 de junio de 1938 establecía la obligación de las empresas, cuando así lo solicitase la mitad del personal obrero, de conceder dos horas para el almuerzo y habilitar un local-comedor adecuado para ello ${ }^{6}$; en enero de 1941 comenzó a legislarse en materia de economatos ${ }^{7}$ y, durante los años

3. Un análisis más amplio en Moreno, Luis; Sarasa, Sebastià. Génesis y desarrollo del Estado del Bienestar en España. [Madrid]: Instituto de Estudios Sociales Avanzados; 1992.

4. Sobre Falange: Payne, Stanley G. Falange. Historia del fascismo español. La Patria, el Pan y la Justicia. Madrid: SARPE; 1985; Payne, Stanley G. Franco y José Antonio. El extraño caso del fascismo español: historia de la Falange y del Movimiento Nacional (1923-1977). Barcelona: Planeta; 1997.

5. Moreno; Sarasa, n. 3, p. 12.

6. Decreto de 8-VI-1938 (BOE 11 Jun 1938); la orden ministerial de 30-VI-1938 (BOE 1 Jun 1938) desarrolla este decreto.

7. Orden ministerial de 30-I-1941 (BOE 31 Ene 1941); una orden ministerial de 6 Abr 1946 (BOE 8-IV-1946) estableció las empresas que, de manera obligatoria, deberían tener economato. 
centrales de la década de 1940, se hacen públicas las primeras medidas legales en materia de viviendas protegidas ${ }^{8}$.

El paternalismo católico desarrolló estos preceptos estatales a través de Acción Social Patronal, una asociación de empresarios españoles, auspiciada por la jerarquía eclesiástica y encuadrada dentro de la organización oficial de Acción Católica Española, encargada de analizar los problemas sociales que se planteaban en el seno de las empresas, siempre en sintonía con las enseñanzas pontificias ${ }^{9}$. El salarial fue uno de los asuntos tratados, con más detalle, por Acción Social Patronal ${ }^{10}$; la organización incitó a los empresarios a que cumplieran con un «salario justo» ${ }^{11} \mathrm{y}$ a que éste fuera suficiente para proporcionar una vida moral y digna al trabajador y a su familia. Estado e Iglesia católica caminaron unidos en materia laboral, al menos durante el primer franquismo ${ }^{12}$.

Con estos mimbres legislativos se construyó el esqueleto paternalista del franquismo autárquico; la doctrina social católica colaboró a sacralizar estas medidas articuladas por el Estado, siempre bajo el prisma anti-socialista y de rechazo hacia la lucha de clases, pero también contra el capitalismo extremo, las grandes sociedades anónimas ${ }^{13}$ y la explotación obrera. En la Carta-Encíclica Rerum Novarum, León XIII reflexionaba sobre la condición obrera, criticaba al socialismo como remedio para acabar con las desigualdades sociales y proponía «el remedio verdadero: la Doctrina católica» ${ }^{14}$.

8. Decreto de 2-III-1944 (BOE 16 Mar 1944) y ley de 17-VII-1946 (BOE 18 Jul 1946).

9. Esta asociación católica incidía en los beneficios que podría tener para el empresario la construcción de viviendas sociales. [Acción Social Patronal]. Las viviendas y la empresa. Madrid: Gráficas Varela; 1952.

10. El decreto de 26-I-1944 (BOE 24 Feb1944) dejaba bien claro el concepto paternalista de salario establecido por el Gobierno.

11. [Acción Social Patronal]. El salario y la empresa. Madrid: Gráficas Orión; 1953, p. 49-52.

12. Algunos autores sostienen que, a partir de 1956, se inició un proceso de disidencia, en el seno de la Iglesia católica española, respecto a ciertos posicionamientos franquistas; éstos optaron por el concepto de «justicia social» frente al planteamiento clásico de «caridad paternalista». Montero García, Feliciano. La Iglesia: de la colaboración a la disidencia (1956-1975). La oposición durante el franquismo. Madrid: Encuentro; 2009; Montero García, Feliciano. La Iglesia y el Tardo-franquismo o el «despegue» de la Iglesia. Historia del Presente. 2007; 10: 3-6.

13. Guerrero, Fernando. El capital en la empresa moderna. Madrid: Propaganda Popular Católica; 1963, p. 10-12.

14. Martín Artajo, Alberto; Cuervo, Máximo. Doctrina social católica de León XIII y Pío XI. Recopilación concordada y profusamente anotada, de los documentos pontificios y sociales sobre la materia.

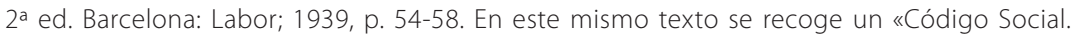
Esbozo de una síntesis social católica», redactado por la Unión Internacional de Estudios Sociales, fundada en Malinas, en 1920, bajo la presidencia del cardenal Mercier (1851-1926), 
No obstante, desde algunos sectores, como la militancia obrera católica española, la doctrina social de la iglesia se constituyó - pese a la oposición de la jerarquía eclesiástica - en un instrumento para denunciar la opresión del régimen franquista y las injusticias del sistema capitalista ${ }^{15}$. Autores como Ignacio Fernández de Castro ${ }^{16}$ entendían que compatibilizar, en España, los intereses católicos con los empresariales era una tarea harto complicada, en particular se mostraba crítico con el colectivo de empresarios católico-capitalistas españoles, «lo más representativo de nuestro catolicismo seglar», sin:

«verdadera conciencia de pecado por su conducta capitalista (...), en la certeza de que completando esta vida económica capitalista con una vida de piedad espiritual de tipo también individualista, están santificando su vida entera (...) Quisiera equivocarme por estos piadosos 'católicos-capitalistas', pero yo, la verdad, no estaría, en su caso demasiado tranquilo» ${ }^{17}$.

¿Hablaba, tal vez, del fundador y dueño de Alter, Juan José Alonso Grijalba?; no necesariamente, aunque -como veremos- el retrato de empresario católico-paternalista español dibujado por Ignacio Fernández de Castro se ajusta, perfectamente, al de nuestro protagonista.

en el que se alude (p. 192), de manera explícita, a la deshumanización a que conducen los sistemas de taylorización del trabajo.

15. Sobre el «catolicismo social», Montero García, Feliciano. La industrialización y sus repercusiones en las formas de vida y en la acción de la Iglesia católica. In: Carmona Fernández, Francisco, coord. Historia del Cristianismo, 4 [El Mundo Contemporáneo]. Salamanca: Trotta; 2010, p. 113-153; Montero García, Feliciano. El primer catolicismo social y la Rerum Novarum en España (1889-1902). Madrid: CSIC; 1983; Montero García, Feliciano. El Movimiento Católico en España. Salamanca: Eudema; 1993. También Fernández Segura, José. La participación de los católicos en el movimiento obrero de Barcelona (1946-1978) [Tesis doctoral, dirigida por Joan Oliver i Puigdomènec]. Barcelona: Departamento de Historia Contemporánea. Universidad de Barcelona; 2005, en particular el epígrafe dedicado a la «Doctrina social de la Iglesia» (p. 122-163).

16. Ignacio Fernández de Castro (1919-2011) sociólogo, abogado laboralista y católico, fue uno de los fundadores del Frente de Liberación Popular (FELIPE) y opositor a la dictadura franquista en la década de 1950. Rogero Anaya, Julio. Recorrido por la vida y la obra de Ignacio Fernández de Castro. Con-ciencia social. 2010; 14: 83-89; Elejabeitia Tavera, Carmen; Gumpert, Leopoldo; Rogero Anaya, Julio; Valero, Alfonso. Ignacio Fernández de Castro y la educación. Cuadernos de pedagogía. 2011; 417: 92-95.

17. Fernández de Castro, Ignacio. Del paternalismo a la justicia social. Madrid: Euramérica; 1956, p. 69-70. 
Nuestro objetivo, en las líneas que siguen, es ahondar en el pensamiento empresarial de Juan José Alonso Grijalba, fundador de los laboratorios Alter y del grupo empresarial que, a través de éste, se consolidó. Utilizaremos para ello los textos de conferencias y ensayos donde éste expuso sus opiniones sobre el modo en que hacía funcionar su empresa, no exentas de datos cuantitativos sobre sus realizaciones. Tras la pérdida de protagonismo del fundador, el modo de entender la organización empresarial fluyó por caminos diferentes, más complejos. Por ello, nuestro trabajo se extiende durante el período en que éste dirigió su grupo empresarial: desde 1939 hasta los inicios de la década de los sesenta.

\section{Juan José Alonso Grijalba (1894-1962)}

Juan José Alonso Grijalba nació el 28 de marzo de 1894, en la localidad riojana de Baños de Río Tobía. Fue el cuarto de los seis hijos - tres varones y tres mujeres - concebidos en el matrimonio formado por Juan Alonso Martínez y Casilda Grijalba Murillo ${ }^{18}$. A los diez años inició sus estudios en el Colegio-Noviciado de los Padres Franciscanos de Nájera, aunque no continuó la carrera eclesiástica, sino la de Farmacia, de la que obtuvo el título de licenciado, por la Universidad de Barcelona, en $1915^{19}$. Su primer trabajo fue el de regente en la farmacia de Villavelayo (La Rioja), y poco después abrió oficina propia en su pueblo natal.

En 1919 contrajo matrimonio con María Samaniego Garnica. La unión tuvo dos hijos: José María (4-6-1920 / 21-2-1984) y Miguel Ángel (31-8-1921 / 3-3-1981); ambos, muy ligados al futuro del grupo empresarial iniciado por la familia y que realizaron estudios de Farmacia.

El pronto fallecimiento de su esposa, en 1923, a consecuencia de un parto fallido, le incitó a trasladarse a Logroño. Se instaló en esta ciudad en 1924 compatibilizando, durante dos años, las labores propias de la oficina de farmacia con las de director de la sucursal del Banco Español de Crédito próximo a su botica. En 1925 contrajo segundas nupcias con María Jesús

18. Ilustrísimo señor don Juan José Alonso Grijalba. 28 Mar 1894 / 12 Mar 1962. Madrid: EMESA; 1962. Biblioteca de la Universidad Pontificia de Comillas, sede Cantoblanco, registro: 269.893. El Índice Histórico Español. 1963; 9 (37): 269, atribuye la autoría a sus hijos; el texto roza el género hagiográfico.

19. La Vanguardia. 25 Ago 1915; 34 (13.358): p. 3. 
Cillero Angulo, con quien tuvo cinco hijos: María del Carmen, José Luis, Francisco Javier, María Jesús y María Teresa ${ }^{20}$.

A partir de su traslado a Logroño comenzó a fraguarse la exitosa andadura empresarial de Alonso Grijalba. A su oficina de farmacia se añadió un laboratorio enológico, independizado como Laboratorio Vitícolo-Enológico de La Rioja, desde el que preparaban abonos minerales y fertilizantes para el campo ${ }^{21}$. En febrero de 1930, durante la dictadura del general Dámaso Berenguer, asumió un puesto de diputado en representación de la Cámara Agrícola, en la nueva Diputación Provincial de Logroño conformada al efecto. Sus actuaciones le sitúan próximo al grupo liberal liderado por Miguel Villanueva Gómez (1852-1931) ${ }^{22}$.

En 1938, en plena Guerra Civil, y tras visitar junto a su sobrino José Alonso Tejada algunas industrias farmacéuticas francesas, suizas y alemanas, tomó la iniciativa de fundar los Laboratorios Alter ${ }^{23}$. Su primer domicilio social quedó en la residencia del fundador. El 14 de julio de 1939 se constituyó, en Logroño, la sociedad Juan José Alonso, S.A., con un capital social de tres millones de pesetas, a la que quedaron vinculados tanto el Laboratorio Vitícola-Enológico de La Rioja como los Laboratorios Alter.

En los primeros meses de posguerra, Alonso Grijalba decidió trasladarse a Madrid ${ }^{24}$. Allí encontró el lugar apropiado para construir la sede definitiva

20. María Jesús Cillero Angulo falleció, en diciembre de 1943, a consecuencia de una pulmonía. Dos de los hijos habidos en este matrimonio, Francisco Javier y María Jesús, fallecieron a temprana edad. José Luis realizó estudios de Economía, María del Carmen contrajo matrimonio con el catedrático de Medicina Manuel Armijo, presidente de Farmabión S. A. tras el fallecimiento de Juan José Alonso Grijalba, y María Teresa con el ingeniero militar Agustín Amoribieta Martí. El texto que su confesor leyó con ocasión de su sepelio tuvo una versión impresa, repartida entre los empleados del Laboratorios Alter: Jiménez Lemuar, Carlos. Doña María Jesús Cillero Angulo de Alonso. 10-I-1894 / 16-XII-1943. Apuntes biográficos por su director espiritual... S.I.: s.n., 1944. Colección particular.

21. El Laboratorio Vitícola-Enológico de La Rioja estuvo inicialmente instalado en la Avenida de Navarra, en Logroño.

22. Navajas Zubeldía, Carlos. La «segunda dictadura» en La Rioja (1930-1931). Berceo. 1996; 130 157-180. Quizás por influencia de su hermano, Buenaventura Alonso Grijalba «diputado provincial por el distrito de Nájera-Torrecilla de Cameros desde 1917 hasta 1923 (...) el verdadero cacique del distrito de Torrecilla» (p. 172).

23. «ALonso, apellido del padre, primer eslabón de la familia, y TEResa, nombre de la hija pequeña, y eslabón último, prestaron sus letras iniciales a la denominación de la empresa que fundó y por la que tanto trabajó don Juan José». Ilustrisimo, n. 18, p 10-11.

24. «Aventura mayúscula la de trasladarse a Madrid. La familia, los amigos, los compañeros, no comprendían por qué levantaba el vuelo de un asentamiento que tan pródigo se le había mostrado. Intentaban disuadirle de un propósito que consideraban en pugna con el tranquilo 
de Laboratorios Alter: un amplio espacio ubicado en la periferia de la ciudad, entonces perteneciente al municipio de Chamartín de la Rosa ${ }^{25}$. La inauguración oficial se produjo el 10 de octubre de 1941 y contó con la presencia del entonces nuncio de Su Santidad en España, monseñor Caetano Cicognani (1881-1962). En 1944 amplió sus instalaciones con un «segundo pabellón» inaugurado por el agustino José López Ortiz (18981992), uno de los confesores elegidos por José María Escrivá de Balaguer en los comienzos de la década de 1940, entonces recién nombrado obispo de Tuy-Vigo ${ }^{26}$. A tenor de la información suministrada por la ficha de encuadramiento en el Sindicato Vertical de Industrias Químicas, además de estas instalaciones, el Laboratorio contaba con locales dedicados a la fabricación en la «Carretera de Zaragoza» y sucursales en varias provincias ${ }^{27}$. En 1940 trabajaban al servicio del Laboratorio ochenta y siete personas, cuarenta y ocho de ellas mujeres ${ }^{28}$.

El fallecimiento de su segunda esposa, en diciembre de 1943, provocó el temporal apartamiento de Alonso Grijalba al frente de Laboratorios Alter, a los que retornó en enero de 1945. A partir de 1947 se sucedieron

y seguro porvenir que allí le esperaba. Pero él no abandonó la visión de su obra futura, y puso pies en Madrid, en 1939». Ilustrísimo, n. 18, p. 15. El Laboratorio Vitícola-Enológico de La Rioja continuó en La Rioja, mientras que la farmacia-droguería de Logroño fue traspasada.

25. En el número 7 de la calle Mateo Inurria, donde aún hoy sigue en funcionamiento.

26. Martín de la Hoz, José Carlos. Un amigo de san Josemaría: José López Ortiz, OSA, obispo e historiador. Studia et Documenta. 2012; 6: 91-121; en gran parte basado en el relato del propio José López Ortiz. Testimonio. In: Badrinas, Benito, ed. Un hombre de Dios. Testimonios sobre el fundador del Opus Dei. Madrid: Palabra; 2002, p. 205-244.

27. La ficha de encuadramiento sindical lleva fecha de diciembre de 1940. El capital social declarado fue de tres millones de pesetas; el cargo de gerente lo ocupaba Juan José Alonso Grijalba, la directora técnica farmacéutica era Ángela Alonso Tejada y el consejo de administración estaba formado por Juan José Alonso Grijalba, Buenaventura Alonso Grijalba, José María Alonso Samaniego, Ángela Alonso Tejada y José Barrado Ruiz. Archivo General de la Administración (AGA), sección Sindicatos, legajo (06) 34/14315.

28. Los varones ocupaban siete puestos técnicos, cinco de ellos de farmacéutico, uno de perito y uno más no especificado; las mujeres se repartían cuatro puestos técnicos, tres de farmacéutico y uno de perito. En el área comercial y de administración trabajaban treinta y ocho personas, veintisiete de ellos varones que se distribuían las tareas de director comercial, dos cajeros, un contable, cuatro mozos de almacén, once visitadores médicos, siete administrativos y uno más sin identificar; los once puestos restantes eran desempeñados por mujeres: una cajera y diez administrativas. En el área de producción estaban empleadas treinta y ocho personas: cinco varones (un contramaestre y cuatro peones especializados) y treinta y tres mujeres, todas ellas con la categoría profesional de peón especializado. AGA, sección Sindicatos, legajo (06) 34/14315. No nos queda constancia de los sueldos que percibían, por lo que no es posible establecer una supuesta diferenciación ligada al sexo. 
sus intervenciones - en muy distintos foros- sobre la puesta en práctica de las enseñanzas pontificias relativas al mundo del trabajo, ejemplarizadas en su quehacer al frente de Laboratorios Alter.

En 1957, cumplidos ya los sesenta años, Alonso Grijalba constituyó el grupo empresarial Alter, en el que se reunieron todas las actividades desarrolladas bajo el paraguas de la sociedad Juan José Alonso S.A. ${ }^{29}$ Además de Laboratorios Alter y el negocio vitivinícola, mantenido a través de Bodegas Riojanas, las actividades del Grupo Alter se extendían al Instituto Terapéutico S.A. (INTESA), construido en 1948 tras la absorción de Laboratorios Bagazgotia; a la distribución de sus productos, mediante Quifasa, una empresa fundada en 1948, con sede en Pamplona y sucursales en Barcelona y Granada; a la fabricación de ampollas inyectables y a la producción de maquinaria de envasado para la industria farmacéutica, tareas desarrolladas desde Envases Industriales S.A. (EISA); y al comercio exterior de sus medicamentos, a través de la firma Intercambios Comerciales S.A., fundada en 1950.

En diciembre de 1954, con el apoyo económico de Ácidos Grasos y Derivados S.A. (Agra S.A.), empresa domiciliada en Lamiaco-Lejona (Vizcaya), organizó Farmabión, un laboratorio dedicado a la fabricación del antibiótico broncopulmonar «Esterloven 500.000», propiedad del laboratorio danés Leo Pharmaceutical Products. En 1957, el binomio Alter-Farmabión llevó a la fundación, en Pamplona, de Penibérica, una empresa dedicada a la producción de antibióticos ${ }^{30}$. Pocos meses después, también en 1957, se gestó Algry S.A., dedicada a la fabricación de materias primas para la industria farmacéutica.

El grupo tuvo intereses en otros sectores empresariales como el cinematográfico, contando con una productora, Productores Cinematográficos

29. Con posterioridad a su primera organización, la estructura del Grupo Alter se vio modificada: en 1972 ampliaría sus intereses vitivinícolas con la fundación de Bodegas Alavesas; meses después, en 1973, se interesó en la fabricación de productos infantiles adquiriendo BebeConfort-Hispanisa; y en 1976 se constituyó ASEMAL, desde la que llevar a cabo la «unidad contable y financiera de todas las empresas del grupo Alter». Por el contrario, también tuvo sus bajas: desde 1984 prescindió de Quifasa, su distribuidora de medicamentos y materias primas. Alter. Historia de una ilusión. 1939-1989. Madrid: Alter; 1991.

30. Sobre la fundación de los laboratorios Farmabión y Penibérica, y su vinculación a la órbita de Alter, Rodríguez Nozal, Raúl. ¿Fortuna o desdicha? La entrada de la penicilina en la España de la autarquía, un nuevo desafío para la farmacia y la industria farmacéutica. In: González Bueno, Antonio et al., eds. Homenaje al Prof. Dr. José Luis Valverde. Granada: SDUHFE/Universidad de Granada; 2011, p. 515-550. 
Unidos S.A. (PROCUSA) ${ }^{31}$, dos distribuidoras, Distribuidores Independientes Peninsular Films Asociados (DIPENFA) y FILMAYER; un conjunto de locales de exhibición sitos en Madrid, agrupados en la empresa EUSA y una financiera especializada en proyectos cinematográficos CIRTE $^{32}$. También en el mundo editorial, representado por Sapientia, una empresa especializada en textos de difusión del pensamiento católico.

Además dispuso de dos filiales farmacéuticas: una portuguesa (Alter SARL, establecida en 1950) y otra dominicana (Alter Dominicana). La última empresa del Grupo Alter en cuya participación Alonso Grijalba tuvo parte activa fue Prominter S.A., nacida en 1960, fruto de la alianza comercial con la estadounidense Sterling-Winthrop, para la comercialización de los productos de la empresa americana en España.

El 12 de marzo de 1962, apenas cinco años después de consolidado su grupo empresarial, Juan José Alonso Grijalba falleció en Madrid, días antes de cumplir los sesenta y ocho años de edad ${ }^{33}$.

\section{Los Laboratorios Alter: un vivero para la materialización de la doctrina social católica en pleno franquismo}

El pensamiento de Alonso Grijalba sobre el mundo laboral y su plasmación en él de la doctrina social de la Iglesia nos ha llegado a través de cuatro textos nacidos con vocación efímera: un par de conferencias

31. La empresa tuvo una amplia participación en su capital de la familia Luca de Tena. No obstante, su primer consejo de administración quedó bajo la presidencia de Juan José Alonso Grijalba. Caparrós Lera, José María. Sobre Procusa (1958-1965), una iniciativa de coproducciones europeas y de cortometrajes. In: Marzal Felici, Javier; Gómez Tarín, Francisco Javier, eds. El productor y la producción en la industria cinematográfica. Actas del II Congreso Internacional sobre Análisis Fílmico. Madrid: Editorial de la Universidad Complutense de Madrid; 2009, p. 143-164.

32. En la junta general de CIRTE S.A., Financiera de Cine, Radio y Televisión, celebrada en julio de 1962, se dedicó «un emocionado recuerdo a don Juan José Alonso Grijalba, recientemente fallecido, cuya labor y entusiasmo fueron indispensables e inapreciables en la fundación y puesta en marcha de CIRTE». La Vanguardia Española. 20 Jul 1962; 78 (29.897): 20.

33. Un relato pormenorizado, casi asfixiante, de los últimos días de Juan José Alonso Grijalba, incluyendo una carta escrita por él y dirigida «A todos mis amigos y colaboradores del Grupo Alter», en: Ilustrísimo, n. 18, p. 64. El sepelio estuvo presidido por Saturnino Rubio y Montiel (1889-1971), riojano de origen, quien ocupaba a la sazón la Diócesis de Osma-Soria, y por Abilio del Campo y de la Bárcena (1908-1980), obispo de Calahorra y La Calzada-Logroño, amigos personales del finado. 
pronunciadas en 1948 y otro par de folletos, en edición numerada, editados en 1952 y 1959.

El primero de estos opúsculos corresponde al contenido de la conferencia pronunciada en el Seminario Diocesano de Vitoria, el 12 de marzo de 1948, bajo el título Aplicación de la doctrina social pontificia en Alter ${ }^{34}$. El texto, de cariz autobiográfico, es una loa a la doctrina social emanada de Quadragesimo Anno (1931) de Pío XI, Divini Redemptoris (1937) de Pío XI o Sertum Laetitiae (1939) de Pío XII, dentro del ambiente político gestado tras la Guerra Civil ${ }^{35}$.

La segunda de las conferencias tuvo lugar ante un auditorio bien distinto, fue pronunciada ante sus propios empleados, reunidos en el VIII Congreso de delegados de Laboratorios Alter, celebrado el 20 de septiembre de $1948^{36}$. El discurso, titulado Cristianismo y empresa, tiene similares estructura y contenido que la conferencia pronunciada apenas unos meses atrás en el Seminario de Vitoria, pero esta vez la dedica «a colaboradores de ideologías afines» ${ }^{37}$.

El tercero es un extenso escrito, editado en 1952, donde, bajo el título de Aspectos sociales de la empresa ${ }^{38}$, resume su pensamiento expresado en la década de 1940 en cuantos foros y lugares pudo ${ }^{39}$.

34. Alonso, Juan José. Aplicación de la doctrina social pontificia en Alter. Vitoria: Social Católica; 1948. Biblioteca Nacional de España (BNE), signatura: VC/2010/69.

35. «... yo quería servirme de la industria, para hacer apostolado social; quería trabajar para gloria de Dios y de la Iglesia. Esto exigía dar a los bienes y a las relaciones laborales, la orientación reclamada por la misma naturaleza de los bienes, e inculcada reiteradamente por la doctrina de la Iglesia». Alonso, n. 34, p. 11.

36. Alonso, Juan José. Cristianismo y empresa. Madrid: Magisterio Español; 1948. Biblioteca de la Universidad Pontificia de Comillas, sede Cantoblanco, registro: 234.371.

37. «unidos en amistosa convivencia para la realización de una obra de utilidad común (...) cuando a la luz de los Ejercicios, habéis visto cuán en consonancia están estos ideales del trabajo cristiano con los fines señalados por Dios a nuestra existencia». Alonso, n. 36, p. 5 y 8.

38. Alonso Grijalba, Juan José. Aspectos sociales de la empresa. Madrid: Magisterio Español; 1952. Biblioteca de la Universidad Complutense de Madrid, Facultad de Trabajo Social, signatura: D658.17ALO. «Su redacción obedece al sencillo propósito de ofrecer un compendio ordenado de ideas (...) que, por encerrar las directrices fundamentales que guían la estructuración de nuestra empresa, nos ha parecido conveniente arrancar de la fugacidad de la palabra hablada para confiarlas a la fijeza de la escritura» (p. 9-10).

39. Sus próximos señalan alguno de estos centros: el Seminario Diocesano de Vitoria, la Asociación de Ingenieros del ICAl, el Congreso Nacional de Hombres de Acción Católica, la sede de Sindicatos en Castellón, el Colegio de la Compañía de Jesús en Barcelona, el Seminario Diocesano de Madrid, el Colegio Español de Roma, la Cámara de Comercio de Santander, la 
El último de los opúsculos fue impreso para conmemorar el vigésimo aniversario de la fundación de Alter, en mayo de $1959^{40}$. El grueso de este folleto conmemorativo se dedicó a las relaciones humanas dentro de la empresa $^{41}$.

En estos cuatro textos se aprecia bien el ideario católico de Alonso Grijalba, para quien la autoridad del empresario era incontestable; su estatus no tenía un origen humano sino divino, se trataba de un don concedido por el Creador que había que obedecer y respetar y, por supuesto, ser agradecido, respetuoso y fiel hacia su persona y la autoridad que encarna. La tarea debía ser sencilla para cualquier trabajador, habida cuenta que -según mantiene en sus escritos- estos «elegidos» estaban adornados con las mejores virtudes para el mando: justicia, equidad, amor al bien común, espíritu insobornable, voluntad, rectitud, entrega total y abnegación puesta al servicio de los demás ${ }^{42}$.

En su opinión, el trabajo era un modo básico de sustento, un medio para huir de la ociosidad y la pereza, una actividad útil que llevaba implícita la «generosidad» del trabajador. Siguiendo su esquema ideológico, el empleado había de ser honesto, fecundo y cumplidor del ordenamiento legal de las leyes de los hombres y, por supuesto, del de Dios:

«Por eso, el cristianismo es la única doctrina que ha sabido encontrar sólidos fundamentos a la dignidad del trabajo. Las demás, aunque parezca que lo exaltan, en realidad lo envilecen ${ }^{43}$.

Universidad Pontificia de Comillas, incluso en un teatro de Santa Cruz de Tenerife. Ilustrísimo, n. 18, p. 25.

40. Relaciones humanas en Alter. Madrid: Alter, Servicio de Publicaciones; 1959. BNE, signatura: VC/4131/42. El folleto, magníficamente editado en hueco-offset, incluye dibujos de Francisco Farreras (n. 1927), fotografías de Juan Pando (1915-1992) y grabados del artista barcelonés V. Tardíu; aun cuando quede atribuido al «Servicio de publicaciones» de la empresa, hubo de contar con el beneplácito de quien fuera su director general.

41. «... para destacar la importancia del hombre, valor máximo a cuyo servicio rendimos todos los demás que integran la Empresa (...) Esta visión humanocéntrica, inspirada en la doctrina social católica y acorde con la cultura de nuestro tiempo, lleva implícita una superación del concepto clásico de la Empresa (...) le cabe la finalidad trascendente de ser creadora de bienestar social» Relaciones, n. 40.

42. Alonso Grijalba, n. 38, p. 106-107.

43. Alonso Grijalba, n. 38, p. 39, 41-42. El entonces obispo de Solsona, Vicente Enrique Tarancón (1907-1994) entendía que el trabajo profesional debería acarrear una serie de deberes u obligaciones del obrero para consigo mismo, la empresa de la que forma parte, sus compañeros de profesión y, por supuesto, la sociedad. Enrique Tarancón, Vicente. Deontología 
En cuanto a la organización del trabajo, el presidente de Alter mostró sus simpatías hacia el «fayolismo», por la importancia que este sistema concedía al factor humano ${ }^{44}$. Para Alonso, la «deshumanización» del trabajo era un grave error al que, de unirse una falta de educación entre los trabajadores, se convertiría en terreno abonado para capitalismos, liberalismos y comunismos. Su solución era la educación cristiana, tanto de tipo religioso-moral, como social, profesional y cultural ${ }^{45}$. Educación, no sólo instrucción, «porque la educación penetra más, llega hasta formar al hombre, de acuerdo con el plan divino, para ponerle en trance de alcanzar su fin último» ${ }^{46}$.

El pensamiento empresarial de Alonso Grijalba no admitía disidencias. Tampoco tenían cabida en él los conflictos sociales y laborales, sobre todo

del empleado y del obrero. In: Morcillo González, Casimiro; Enrique Tarancón, Vicente. Moral profesional: del empresario y del obrero. Madrid: Acción Social Patronal; 1956, p. 41-96 (52).

44. El término «fayolismo» alude a la doctrina sobre la administración del trabajo propuesta por Henri Fayol (1841-1925), centrada en la dirección empresarial a modo de «escuela de jefes». Fayol defendía la división del trabajo y la especialización, la disciplina, la necesidad de que cada empleado reconociera a un solo jefe —imbuido de la autoridad y responsabilidad inherente a su estatus-, la subordinación del interés particular a un bien general perfectamente definido, el respeto al orden y a la cadena de mando, el sentido de equidad, estabilidad y remuneración justa del trabajador, y la conveniencia de que los empleados estuvieran unidos y suficientemente estimulados para que pudieran aportar iniciativas de interés para la empresa. Mientras que Henri Fayol orientó sus esfuerzos hacia la estructura general de la administración empresarial, Frederik Winslow Taylor (1856-1915) se centró más en optimizar el método y buscar las herramientas de trabajo adecuadas para conseguir una mayor eficacia, aunque fuera en detrimento de los trabajadores. Además de estas dos doctrinas, «fayolismo» y «taylorismo», en el siglo XX apareció el «fordismo», un sistema que defendía la producción en serie a partir de la adecuada transformación de los procedimientos industriales, y que también se caracterizaba por la especialización (o división del trabajo) y la reducción de costos, con el consiguiente aumento de mercancías en circulación y la llegada de productos a nuevos grupos sociales (subalternos e, incluso, asalariados con mayor poder adquisitivo gracias al poder de mediación de los sindicatos). Chiavenato, Idalberto. Introducción a la Teoría General de la Administración. 7a ed. México D.F.: McGraw-Hill Interamericana; 2006; Coriat, Benjamín. El taller y el cronómetro. Ensayo sobre el taylorismo, el fordismo y la producción en masa. Madrid: Siglo XXI; 1982; Fayol, Henri. Administración Industrial y General. Coordinación - Control - Previsión - Organización - Mando. 14a ed. Buenos Aires: El Ateneo; 1987; Taylor, Frederik W. Principios de la Administración Científica. 10a ed. Buenos Aires: El Ateneo; 1987

45. «La gran preocupación del patrono ha de ser, pues, hacer a los que de él dependan más felices, más cultos, más morales y espirituales; y su gran tarea organizar una empresa donde la cultura, la moral y la religión sean los cimientos estables de la convivencia». Alonso Grijalba, n. 38, p. 31.

46. Alonso Grijalba, n. 38, p. 80. 
si venían de la mano del movimiento obrero. Su modelo, rebosante de caridad y hermandad, se postulaba como alternativa al socialismo y a la lucha de clases, como «única fórmula capaz de aunar los antagonismos que hoy dividen al capital y al trabajo» ${ }^{47}$.

No puede decirse que el ideario social católico de Alonso Grijalba, que con tanto empeño predicó, cayera en saco roto. Su empresa se convirtió en modelo de ideario cristiano y sus actuaciones concretas, además de coherentes con esta ideología, fueron numerosas. En 1962, Sabino ÁlvarezGendín, catedrático de Derecho Administrativo, magistrado del Tribunal Supremo y rector de la Universidad de Oviedo, citaba media docena de ejemplos de gestión económica laboral de la empresa acorde con la doctrina social católica, entre ellos señalaba:

«La empresa Alter, S.A., de productos farmacológicos, con laboratorios instalados en Madrid, tiene 400 productores que disfrutan retribuciones de más del 50 al 100 por 100 que lo marcado por la Ley; salario familiar extraordinario, primas y participación en los beneficios, cuya cifra mínima obligatoria se incrementa conjugando la categoría profesional del interesado, su rendimiento laboral y sus méritos. Últimamente se ha implantado un régimen de acciones vinculadas al trabajo, que hace a los obreros accionistas de la empresa, amén de otras muchas ventajas sociales» ${ }^{48}$.

La materialización de los postulados sostenidos por Alonso Grijalba se produjo en tres frentes: el económico, el cultural-recreativo y el moralreligioso.

47. «Sin la doctrina de la Iglesia, sin sus principios morales, el arduo problema social se agrava (...) El odio hace su funesta aparición, la caridad se extingue, surge la lucha de clases, de la que, aferrados los combatientes a su verdad parcial, solamente se derivan desastres para la sociedad». Alonso Grijalba, n. 38, p. 18 y 33. Una opinión muy en sintonía con los planteamientos defendidos por el arzobispo de Zaragoza, Casimiro Morcillo González. Deontología del empresario. In: Morcillo González, Casimiro; Enrique Tarancón, Vicente. Moral profesional: del empresario y del obrero. Madrid: Acción Social Patronal; 1956, p. 13.

48. Álvarez-Gendin, Sabino. La participación laboral en la economía y administración de las empresas y la doctrina de los pontífices. Madrid: Imprenta Nacional del BOE; 1962, p. 56. Guerrero, n. 13, también defiende la participación del obrero en el accionariado de empresas medianas y pequeñas, a menudo familiares, siempre desde la óptica de la doctrina social católica. 


\subsection{El componente económico}

En el ámbito económico, el paternalismo empresarial postulado por Juan José Alonso se organizó desde dos grandes frentes: los beneficios directos (salarios, pluses, y participación accionarial) y los indirectos (viviendas, comedores, caja de previsión, servicio médico, etc.).

Según Alonso Grijalba, en la década de 1940 los salarios en Alter fueron establecidos con una mejora general del $20 \%$ respecto de los sueldos base marcados por el Gobierno ${ }^{49}$, pero tal incremento se ejecutaba en razón de la situación familiar del empleado. Los casados percibirían aumentos lineales del 10\%, mientras que los solteros habrían de conformarse con ver cómo se ingresaba ese porcentaje en una cartilla de ahorro prematrimonial hasta que cumplieran 30 años. Por cada hijo también se recibirían aumentos, independientes de los establecidos por el Estado para cargas familiares. La participación en beneficios se hacía a través de pagas extraordinarias - seis a finales de la década de 1940-. En 1948, un obrero de Laboratorios Alter, casado y con dos hijos, recibía un sueldo diario de 35 pesetas ${ }^{50}$.

Un sueldo, como reconocía el propio Alonso, escaso para mantener una familia obrera en Madrid. A partir del 1 de enero de 1948, por acuerdo del Consejo de Administración y tras ocho días «en perfecto retiro», Alter implantó el «salario familiar», tal y como venía siendo propugnado por la doctrina papal ${ }^{51}$. La tabla 1 muestra el «salario familiar» para técnicos y jefes y para obreros y subalternos, tal como recogió el propio Alonso Grijalba en $1948^{52}$, comparados con el coste de la vida reflejado en el Anuario Estadístico de España de ese mismo año ${ }^{53}$.

49. En 1959 la empresa refería retribuciones económicas superiores al 180\% de las legalmente establecidas por el Gobierno. Relaciones, n. 40.

50. Un estudio sobre salarios reales de la industria española (1936-1941), realizado por el Consejo de la Economía Nacional, en Martínez Mesa, Francisco José. Una aproximación al nivel de vida obrero en la España de la Autarquía: la encuesta sobre salarios del Consejo de Economía Nacional. In: Castillo Alonso, Santiago, coord. El trabajo a través de la historia. Madrid: Asociación de Historia Social / Secretaría de Formación Confederal -UGT / Centro de Estudios Históricos; 1996, p. 509-515.

51. En términos conceptualmente similares a los empleados por el —entonces- arzobispo de Zaragoza. Morcillo, n. 47, p. 21.

52. Alonso, n. 34, p. 21. «No hay para que describir los demás grupos de la escala, conocidos que no son los extremos, pues todos guardan la proporción debida a su posición social».

53. Instituto Nacional de Estadística. Anuario Estadístico de España. 1949; 24: 632-637. Sobre la poca fiabilidad de los datos salariales disponibles, la complejidad del sistema de retribuciones 
Tabla 1. Salarios familiares de los trabajadores de Alter en 1948

\begin{tabular}{|c|c|c|c|}
\hline Salario familiar & \multicolumn{2}{|c|}{ Grupo Alter } & Coste de la vida \\
\hline & Técnicos y jefes & Obreros & \\
\hline Vivienda & 600 pesetas & 250 pesetas & 135,2 pesetas \\
\hline Alimentación & 800 pesetas & 800 pesetas & 557,6 pesetas \\
\hline Vestuario / Vestido & 400 pesetas & 100 pesetas & 554 pesetas \\
\hline $\begin{array}{l}\text { Servicio doméstico / } \\
\text { Gastos de casa }\end{array}$ & 500 pesetas & - & 373 pesetas \\
\hline $\begin{array}{l}\text { Varios / } \\
\text { Gastos generales }\end{array}$ & 400 pesetas & 100 pesetas & 289,1 pesetas \\
\hline Por cada hijo & 1.050 pesetas & 690 pesetas & - \\
\hline Total & 3.750 pesetas $/ \mathrm{mes}$ & 1.940 pesetas $/$ mes & $1.908,9$ pesetas/mes \\
\hline
\end{tabular}

Fuente: Elaboración propia a partir de Alonso, Juan José. Aplicación de la doctrina social pontificia en Alter. Vitoria: Social Católica; $1948^{54}$.

Las empleadas que, una vez casadas, decidieran abandonar la empresa serían compensadas, además de con una paga por matrimonio, con tantas pagas mensuales como años llevaran de servicio en la empresa. Éstas eran también las condiciones establecidas para aquéllas que decidían aceptar el estado religioso ${ }^{55}$. Por nacimiento de niño se concedía media paga

durante este período y las deficiencias de los Anuarios Estadísticos de España como fuente para este tipo de estudios. Vilar Rodríguez, Margarita. El precio del trabajo industrial en las primeras décadas del franquismo (1936-1963): las limitaciones de las fuentes estadísticas. Madrid: Fundación Primero de Mayo; 2005. Carmen Benito del Pozo señala la carencia de fuentes informativas empresariales para el análisis de los salarios; nuestros datos tienen, precisamente, este origen empresarial. Benito del Pozo, Carmen. El salario obrero en los años 50 y la crisis del modelo retributivo autárquico. Cuadernos Republicanos. 1993; 16: $45-60$.

54. Como elemento comparativo utilizamos la media de los índices mensuales proporcionados por el Anuario Estadístico de España para la ciudad de Madrid, correspondientes al año 1948. Nuestros datos se establecen para familias con dos hijos menores de catorce años. Los conceptos indicados por Alonso Grijalba y el Anuario Estadístico de España no responden, en algunas ocasiones, a un mismo criterio estricto; lo indicamos separando mediante una barra oblicua el criterio de estimación señalado por cada una de las fuentes. En primer lugar figura el establecido por Alonso Grijalba.

55. En palabras de Alonso Grijalba, «gracias a Dios, las rosas perfumadas de vocaciones, florecen abundosas en el jardín de Alter». Alonso, n. 34, p. 24. 
extraordinaria y, en caso de que fuera el primogénito, la «Hermandad de Alter» regalaba una canastilla. Cuando el hijo de cualquier empleado del laboratorio hacía su primera comunión, la gratificación era de un cuarto de paga para el progenitor ${ }^{56}$.

Dada la escasez de alimentos en la posguerra y los precios, altos y fluctuantes, que adquirían éstos en el mercado clandestino, Alter optó en la década de 1940 por poner en marcha un economato y comedores para sus empleados. En principio se estableció un comedor para hombres y otro para mujeres, donde se podía acceder a un menú que, según los técnicos médicos de la empresa, era de calidad y suficientemente calórico. Según los cálculos de Alonso, el gasto de la empresa en comedores -más allá de edificios y servicios generales- era de cien mil pesetas anuales ${ }^{57}$. El economato, establecido en 1948, acabó sustituyendo al inicial «plus de carestía de vida» que venían recibiendo los trabajadores de Alter. El economato permitía un racionamiento adicional al marcado por el Gobierno: en 1948 estaba establecido en ocho kilos de legumbres y uno de aceite, por persona y mes ${ }^{58}$. El mantenimiento del economato suponía, a fines de la década de 1940, unas doscientas mil pesetas anuales, aportadas por la empresa.

Otra de las ventajas económicas de la que podían disfrutar los empleados de Alter era un «fondo de préstamos», instituido con el fin de facilitar la adquisición de muebles y enseres domésticos por parte de los empleados. Las cantidades solicitadas no podían sobrepasar el 25\% del salario profesional anual. La Caja de previsión laboral de Alter nació con el objetivo de mejorar las prestaciones otorgadas por el Montepío oficial de Industrias Químicas ${ }^{59}$. La tabla Figura 2 muestra sus principales beneficios.

56. Estas gratificaciones, aplicadas durante los años cuarenta, fueron sensiblemente rebajadas años después; a finales de la década de 1950. Relaciones, n. 40.

57. El primer comedor para empleados, conocido como «La Casina», fue establecido en 1943. Relaciones, n. 40.

58. Alonso, n. 34, p. 18-19.

59. Aprobada por orden del Ministerio de Trabajo de 30 de diciembre de 1949. BOE 24 Ene 1950. 
Tabla 2. Tabla comparativa de pensiones ofrecidas por el Laboratorio Alter

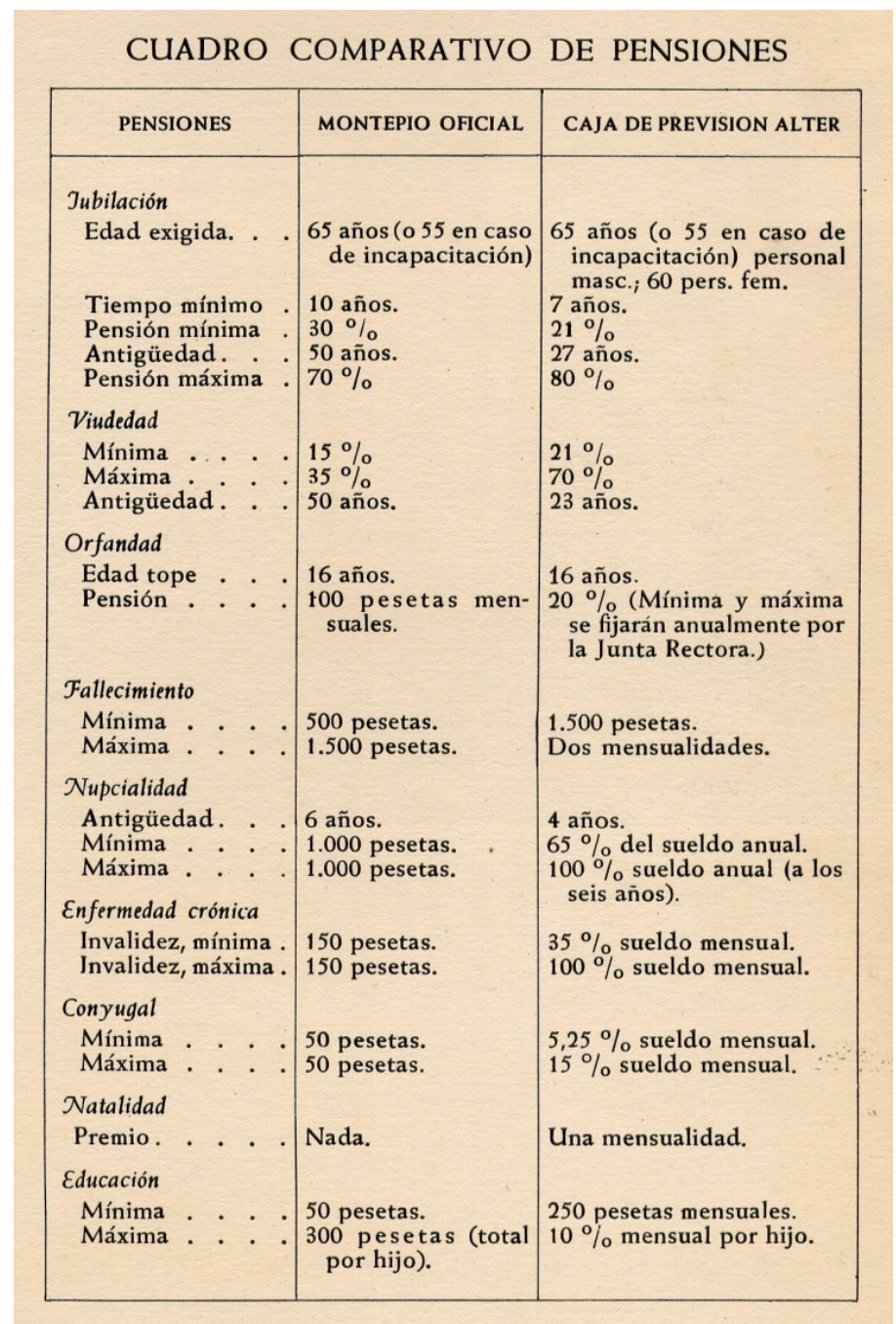

Fuente: Relaciones humanas en Alter. Madrid: Alter, Servicio de Publicaciones; 1959.

En 1957 la empresa emitió una serie de acciones, la serie B de «Acciones de capital vinculadas al trabajo», que fue ofrecida a los empleados que tuvieran una determinada antigüedad «y una sindicación prudente que asegure la permanencia de dichas acciones entre colaboradores directos 
de la empresa ${ }^{60}$. El número máximo del nominal que podía tener cada empleado resultaba de multiplicar su sueldo base por el número de años de servicio.

En caso de enfermedad, los empleados y empleadas de Alter podían contar con médico de empresa, enfermera y con «visitadoras de enfermos» de la «Hermandad de Alter». Este servicio médico también tenía cobertura para familiares, incluyendo la asistencia en partos. Los medicamentos recetados, siempre que fueran fabricados por el laboratorio, eran gratuitos para sus empleados, al igual que todos los inyectables. No existían deducciones por faltas al trabajo, siempre que fuera por enfermedad y, en caso de baja, la empresa abonaba la diferencia entre la indemnización concedida por el Seguro Obligatorio de Enfermedad (50\%) y el total del sueldo.

Por fallecimiento de un empleado, la empresa otorgaba a sus deudos la indemnización que le correspondiera en función de lo establecido por la Caja de previsión laboral. Además, se celebraba la preceptiva misa en la capilla de la empresa, a la que preceptivamente acudía todo el personal ${ }^{61}$.

Alter también dispuso de edificios para sus empleados. La primera residencia para trabajadores solteros se inauguró en 1943; hacia 1950 la empresa contaba con tres bloques de viviendas sitas en las calles Francisco Suárez y Abedul, en Madrid, en total dieciocho pisos; y, por esas fechas, se había iniciado la construcción de otras cuarenta y ocho viviendas ${ }^{62}$. También existían subvenciones dirigidas al alquiler, además de pisos alquilados, a nombre de la empresa, cedidos a su personal.

\subsection{El componente cultural-recreativo}

En el ámbito de la formación, Alter puso a disposición de su personal, desde 1943, diferentes actividades docentes segregadas por sexo y categoría laboral

\footnotetext{
60. Relaciones, n. 40 .

61. En estas situaciones es donde el paternalismo católico de Alonso se manifestaba sin tapujos: «Yo os puedo decir, por la experiencia que de ello tengo, que nada hay que tanto acerque a patronos y obreros, como esta efusión de caridad para con ellos. Estoy persuadido que no les ganaremos con el solo dinero (...) lo que les mueve y estrecha los lazos de unión y amor cristiano con los directivos, son las muestras sinceras de amor, de interés por su familia y sus cosas; los pequeños sacrificios hechos por aliviarles sus penas. No lo dudéis; los obreros, los humildes, tienen mucho corazón». Alonso, n. 34, p. 25.

62. Relaciones, n. 40.
} 
del empleado. Los obreros recibían formación en cultura general, sociología y religión; los subalternos y oficinistas, en comercio y religión; los técnicos y jefes en idiomas, sociología y dogma; y el propio consejo de administración asistía a cursos de moral profesional y ascética. Las enseñanzas específicas para mujeres también quedaban organizadas en función de su puesto profesional. Podían formarse en corte y confección, religión y ascética, cálculo y contabilidad, mecanografía, idiomas, puericultura y enfermería, música y canto ${ }^{63}$. Estas clases de formación se repartían en varios días de la semana, en horas de trabajo y, también, utilizando el tiempo libre del trabajador.

Si la formación era asunto de vital importancia para los intereses católicos de la empresa, aún lo era más la educación, por cuanto «para que la formación sea perfecta, uniforme y eficiente, es necesario comenzarla desde el principio», esto es, desde la enseñanza primaria. En el hogar infantil de Alter, germen del futuro colegio «Juan José Alonso», compartían espacio, bajo la dirección de una profesora teresiana, los hijos de cuantos empleados componían la empresa, «en verdadera hermandad, pues desde mis nietos hasta los de las mujeres de la limpieza viven unidos; reciben esmerada formación desde los dos años; y comen y meriendan juntos» ${ }^{64}$. El centro impartía enseñanzas desde el grado de maternal (2-4 años), hasta el preparatorio de ingreso (8-10 años), con aulas independientes, separadas por sexos, excepto en maternal. Este centro de enseñanza infantil disponía de edificio propio, con jardín, piscina, frontón y recinto recreativo cubierto. En el curso 1958/59 entró en funcionamiento un centro de enseñanza media financiado por Alter, en el que, además de las enseñanzas propias del bachillerato, se impartían clases de idiomas. Para aquellos hijos de empleados que no pudieran asistir a estos centros educativos, la empresa subvencionaba el $75 \%$ de los gastos en libros y matrículas y, en el caso de que estuvieran en régimen de media pensión, con el 50\% del importe correspondiente a esta partida. Eso sí, para disfrutar de las subvenciones se exigía «un mínimo de aprovechamiento».

63. En la línea del modelo de educación de la mujer durante el franquismo, Roca i Girona, Jordi. De la pureza a la maternidad. La construcción del género femenino en la posguerra española. Madrid: Ministerio de y Cultura; 1996; Roca i Girona, Jordi. Esposa y madre a la vez: construcción y negociación del modelo de mujer ideal bajo el (primer) franquismo. In: Nielfa Cristóbal, Gloria (ed.). Mujeres y hombres en la España franquista: sociedad, economía, política, cultura. Madrid: Instituto de Investigaciones Feministas; 2003, p. 45-65.

64. Alonso, n. 34, p. 28-29. 
También se trató de facilitar los estudios universitarios al personal que, con buenas calificaciones en el bachillerato, había tenido que dejar los estudios por motivos laborales. En los primeros años de funcionamiento de Alter, los empleados que se encontraban en esta situación pudieron disponer de la tarde libre y se les facilitaba la adquisición de libros y matrículas. Sin embargo, pronto se hizo evidente que el sistema no permitía compatibilizar la actividad laboral con la universitaria. Ante esta situación, se optó por la fundación de Cor Jesu, una residencia donde jóvenes con brillantes calificaciones en el bachillerato y en la universidad eran formados como empresarios de elite para provecho de la industria española. Una verdadera «forja de apóstoles de la acción social cristiana». Hacia 1948 contaba con treinta y seis residentes, todos ellos becados en sus estudios universitarios y convenientemente adoctrinados con clases adicionales de Filosofía, Moral, Teología y Ascética. Algo así como una especie de alevines de empresariosmonje, que simultaneaban una cualificada formación profesional con misas, rosarios, meditaciones y lecturas espirituales, en definitiva, clones del propio Juan José Alonso Grijalba ${ }^{65}$.

Todo era en Alter un ejemplo de sociedad autárquica, en la que sus integrantes podían vivir sin apenas salir de sus instalaciones: trabajo, comida, vivienda, financiación, atención médica, formación y educación e, incluso, actividades deportivas y de ocio. En lo que concierne a este apartado, Alter contó, desde 1956, con tres piscinas (una para hombres, otra para mujeres y una tercera infantil, situada en el centro de enseñanza), tres frontones (uno para cada sexo y otro más en el hogar infantil) y campo de deportes. Y cine en el salón de actos de la empresa ${ }^{66}$, donde además de impartirse conferencias de temática religiosa y profesional y de dar cabida al coro del

65. Alonso, n. 34, p. 37-38. En la misma línea ideológica defendida por Fernando Martín-Sánchez Juliá (1899-1970), presidente de la Asociación Católica Nacional de Propagandistas entre 1935 y 1953: «La Asociación Católica Nacional de Propagandistas quiere ser una obra de formación y conservación de una minoría selecta con capacidad de dirección (...) una minoría selecta de elementos directores al servicio de la Iglesia». Martín-Sánchez Juliá, Fernando. Ideas claras. Reflexiones de un español. Madrid: Gráficas Nebrija; 1959, p. 298. El centro quedó bajo la tutela de la Compañía de Jesús y, en vida de Alonso Grijalba, se transformó en Colegio Mayor. Ilustrísimo, n. 18, p. 25.

66. El «Pabellón social» de Alter, que albergó los servicios de comedor y salón de actos, fue construido en 1947. Sobre la utilización del cine para el adoctrinamiento, Sanz Ferreruela, Fernando. El cine como catequesis durante el franquismo: la serie de documentales de Magíster, S.A. (1945-1947). Artigrama. 2006; 21: 769-796. 
Laboratorio, se proyectaban periódicamente películas «técnica y moralmente seleccionadas» ${ }^{67}$.

Las vacaciones también fueron objeto de organización, subvención y control por parte del Laboratorio. Durante los años cuarenta se ofrecían casas en las playas del Cantábrico para estancias de 16 días por no más de 100 pesetas por familia obrera - única categoría sujeta a ayuda-. El gasto de Alter en este apartado ascendía a las 50.000 pesetas anuales, un promedio de 1.000 pesetas por familia. Durante los años cincuenta ya se organizaban «tandas de veraneo en playas y montaña, distribuidas por todo el territorio nacional, procurando variar cada año para que puedan ser conocidos lugares diferentes» 68 .

\subsection{El componente moral-religioso}

El espíritu de unión familiar y de confraternización que pretendía Alonso Grijalba para sus empleados se sostenía sobre el pilar de las actividades y celebraciones de orden religioso:

«La Empresa cristiana, tiene que ser casa, donde se gane el hombre decorosamente su vida; pero también centro, donde se instruya y se forme, templo, donde se santifique, hogar donde haya aliciente para la virtud. Así queremos que sea Alter» ${ }^{69}$.

El fundador de Alter tuvo como director espiritual -al menos durante los años 1940- al jesuita Manuel Sánchez Robles ${ }^{70}$. Acostumbraba a practicar la comunión diaria, cada jornada utilizaba entre dos y tres horas «en dar gracias

67. En 1948 Juan José Alonso aludía al cine como posible actuación de futuro. Alonso, n. 34, p. 36.

68. Relaciones, n. 40.

69. Alonso, n. 34, p. 35.

70. Probablemente Manuel Sánchez-Robles Vidal (c. 1881-1966), licenciado en Ciencias Naturales por la Universidad de Madrid, rector del Colegio de los Jesuitas de Sevilla, rector del Instituto Católico de Artes e Industrias [ICAI] (1921-1924), director de la Provincia jesuítica de Toledo y «Rector del Filosofado» en Bélgica (1935-1937); tras la Guerra Civil fue profesor y rector de la Facultad de Filosofía de la Compañía de Jesús establecida en Chamartín de la Rosa (Madrid) y rector del Colegio-Noviciado de San Estanislao en Aranjuez; tras residir en la comunidad del Colegio de San José, en Villafranca de los Barros (Badajoz), pasó al Colegio Noviciado de Aranjuez, donde falleció a los 85 años. Sanz de Diego, Rafael María. ICAI-ICADE; un centro educativo complejo y plural. In: Gil Coria, Eusebio, ed. La Universidad Pontificia de Comillas: cien años de historia (1892-1992). Madrid: Universidad Pontificia de Comillas; 1993, p. 153-278. 
al Señor»y, sólo después, comenzaba a despachar los asuntos económicos y laborales. En 1957, el papa Pío XII le nombró caballero comendador de la orden de San Silvestre Papa ${ }^{71}$.

Juan José Alonso se muestra partidario de las ideas preconizadas por Riccardo Lombardi (1908-1979), jesuita, fundador del Movimiento por un Mundo Mejor (MMM), y colaboró en la difusión de sus ideas en España. El propio R. Lombardi visitó las instalaciones de Laboratorios Alter en $1957^{72}$.

Por supuesto, Laboratorios Alter dispuso de una capilla entre sus instalaciones, constituida en «oratorio semipúblico» gracias a las correspondientes licencias eclesiásticas. En ella se celebraba misa diaria una hora antes de comenzar la jornada de trabajo para que, después, diera tiempo a desayunar. En opinión de Carlos Jiménez Lemuar, cura párroco de San Miguel (Chamartín de la Rosa, Madrid) y encargado del culto en Alter en la década de 1940, la empresa era modelo para todo el colectivo católico y sus actuaciones en el ámbito religioso llegaban más allá de la misa diaria ${ }^{73}$.

\section{Corolario}

El estudio de las relaciones laborales en la industria farmacéutica, durante el franquismo, se plantea como un tema novedoso y poliédrico. Nuestro análisis se ha ceñido a la visión empresarial de Juan José Alonso Grijalba basada en su «paternalismo católico», sin duda un elemento representativo de la política del régimen aun cuando todavía no podamos señalar si se trata de la visión predominante o, tan solo, de una tendencia más o menos

71. Ilustrísimo, n. 18, p. 37, 47, 54.

72. No fue ésta su única colaboración económica prestada; en 1956 co-financió el establecimiento del Carmelo de Villagarcía de Campos, en respuesta a una solicitud de Jesús del Portillo S. J. llustrísimo, n. 18, p. 36.

73. «Laboratorios Alter es una empresa eminentemente cristiana que está realizando una labor profundamente católico-social (...) las visitas de enfermos, la Fiesta de la Madre, el Retiro espiritual del primer viernes, la Cabalgata de los Reyes en colaboración con la Parroquia, el Evangelio de los sábados, todo esto son (...) índices — algunos solamente- de la actuación religioso-social de Alter». Jiménez, n. 20, p. 6. Éstos eran los actos religiosos realizados por Alter en la década de 1950. En los años cuarenta parece que la actividad religiosa era aún más intensa. Alonso, n. 34, p. 34-35. Además de estas actividades periódicas, se realizaron algunos eventos especiales, como sendas peregrinaciones a Roma: una en 1950, con motivo de la celebración del Año Santo, y la otra en 1957, donde más de quinientas personas de Alter fueron recibidas por el Papa en su residencia de Castelgandolfo. Ilustrísimo, n. 18, p. 37. 
vigorosa. Los modos de actuación de Juan José Alonso en Alter fueron los del «patronazgo patriarcal»y, por mucho que tratara de desmentirlo ${ }^{74}-\mathrm{o}$ precisamente por eso-, su aspiración parece ser la de convertir Alter en una fábrica de «obreros-penitentes» ${ }^{75}$, una «verdadera comunidad cristiana de trabajo» ${ }^{76}$ bajo las bases programáticas del humanismo católico ${ }^{77}$. Es posible que esta visión, claramente vinculada con la doctrina social de la Iglesia, condujera a una sinergia empresarial entre distintos intereses económicos que aún no podemos bosquejar.

Los textos de Alonso Grijalba no sólo manifiestan un modelo teórico. Sus ideas fueron aplicadas en todas las empresas del Grupo Alter. Desconocemos si este modelo se implantó en otras industrias farmacéuticas durante el franquismo. Las actuaciones de carácter católico-paternalista desarrolladas por Alonso Grijalba guardan estrechas similitudes con las establecidas por Juan Antonio Suanzes Fernández en el Instituto Nacional de Industria, cuyos subsidios de carácter familiar han sido bien analizados ${ }^{78}$. Hasta qué extremo esta conjunción de intereses entre empresas públicas y privadas fue una situación obligada por el Estado o una opción personal del empresario, precisa aún de un estudio comparado al que esperamos contribuir con los testimonios que presentamos en este trabajo.

El mundo laboral en la industria farmacéutica del franquismo se nos presenta aún como un problema virgen; no tenemos información contrastada sobre los movimientos obreros, sobre la conflictividad laboral, sobre la situación sindical real, ni sobre el rol jugado por la mujer en este sector, que se nos parece revelar como de mayor presencia en puestos de responsabilidad que en otras actividades industriales; no son pocas las mujeres que accedieron a la dirección técnica de un laboratorio durante estos años ni tampoco las que formaron parte de las sociedades propietarias de éstos aunque, desde luego, siguen suponiendo franca minoría frente a los

74. «No queremos hacer un convento, como muchos nos dicen; pero sí queremos acabar con los equívocos y mixtificaciones de cristianos de nombre y paganos o mundanos materialistas en la realidad. Lo que somos: cristianos, seámoslo de verdad y con valentía y sinceridad. Eso queremos». Alonso, n. 36, p. 29-30.

75. Sierra Álvarez, n. 2, p. 103-104.

76. Guerrero, n. 13, p. 12.

77. «No ha de ser el hombre para la economía ni para el progreso o la producción, ni para otro hombre, el Estado o la raza, sino que todo ha de subordinarse al bien del hombre mismo, y éste sólo a Dios». Alonso Grijalba, n. 38, p. 20-21.

78. Mora, José Babiano. Paternalismo industrial y disciplina fabril en España (1938-1958). Madrid: Consejo Económico y Social de España, 1998. 
varones. No cabe duda que es mucho el camino que queda por andar para desbrozar el funcionamiento laboral de la industria farmacéutica durante el franquismo, que probablemente tenga, por sus especiales características, matices propios. 